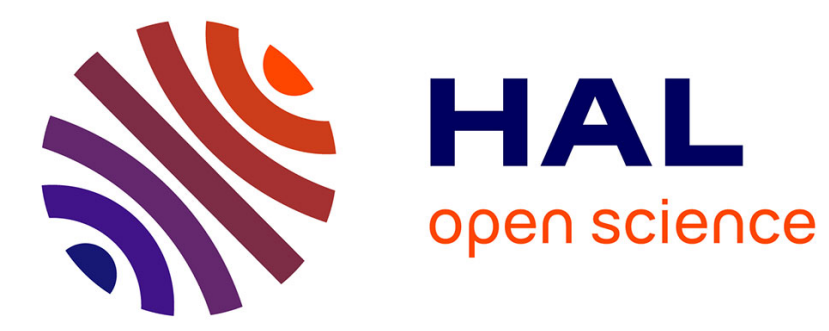

\title{
La peur ultime dans le théâtre de Shakespeare Jean-Louis Claret
}

\section{- To cite this version:}

Jean-Louis Claret. La peur ultime dans le théâtre de Shakespeare. Actes des congrès de la Société française Shakespeare, 2018, 36, 10.4000/shakespeare.4059 . hal-01726729

\section{HAL Id: hal-01726729 \\ https://hal-amu.archives-ouvertes.fr/hal-01726729}

Submitted on 8 Mar 2018

HAL is a multi-disciplinary open access archive for the deposit and dissemination of scientific research documents, whether they are published or not. The documents may come from teaching and research institutions in France or abroad, or from public or private research centers.
L'archive ouverte pluridisciplinaire HAL, est destinée au dépôt et à la diffusion de documents scientifiques de niveau recherche, publiés ou non, émanant des établissements d'enseignement et de recherche français ou étrangers, des laboratoires publics ou privés. 


\section{La peur ultime dans le théâtre de Shakespeare}

Jean-Louis Claret

\section{Q OpenEdition \\ Journals}

\section{Édition électronique}

URL : http://journals.openedition.org/shakespeare/4059

DOI : 10.4000/shakespeare.4059

ISSN : 2271-6424

Éditeur

Société Française Shakespeare

\section{Référence électronique}

Jean-Louis Claret, "La peur ultime dans le théâtre de Shakespeare », Actes des congrès de la Société française Shakespeare [En ligne], 36 | 2018, mis en ligne le 19 février 2018, consulté le 08 mars 2018. URL : http://journals.openedition.org/shakespeare/4059; DOI : 10.4000/shakespeare.4059

Ce document a été généré automatiquement le 8 mars 2018.

(c) SFS 


\title{
La peur ultime dans le théâtre de Shakespeare
}

\author{
Jean-Louis Claret
}

Parler de peur ultime revient à adopter une approche introspective. Ce qui fait le plus peur est en effet lié à l'histoire personnelle de chacun, à son intimité la plus secrète et le révéler revient à se mettre en danger. Mais la peur ultime a pu, au fil du temps, prendre une dimension collective. À l'époque de Shakespeare, les représentations mentale et picturale du Diable et de l'enfer ont atteint un tel degré de développement que ces deux terreurs ont pu occuper dans les esprits toute la place réservée à la peur. L'apparence du prince des ténèbres avait été déterminée par un lent processus dont Robert Muchembled situe le commencement au XIIème siècle. À la Renaissance, il était partout : on pouvait le croiser au coin d'une rue, dans une taverne, sur une scène de théâtre et même dans la pénombre d'un monastère. La peur qu'il inspirait pouvait alors se substituer aux peurs individuelles. Je vais m'attacher_à cette créature redoutable qui a nourri les esprits crédules pendant des siècles et voir comment Shakespeare a su décupler la peur que ce personnage inspirait à ses contemporains. Associer le Diable à la mer, que Jean Delumeau situe à la toute première place dans le palmarès des peurs, est une tentation à laquelle le dramaturge élisabéthain n'a pas eu peur de succomber. Je vais donc m'intéresser tout d'abord à la peur du Diable et de l'enfer pour ensuite me concentrer sur la peur de la mer. Ce travail nous permettra enfin de proposer une lecture originale de Richard III, ${ }^{1}$ pièce dans laquelle un prisonnier, Clarence, raconte le rêve qu'il vient de faire et au cours duquel il décrit son arrivée en enfer après s'être noyé.

\section{Le Diable}

2 Commençons par le Diable. À la fin du XVI siècle, il n'est plus nécessaire de le nommer précisément sur la scène de théâtre. Lorsque Richmond parle de l'ennemi de Dieu (« God's enemy » V.iii.252 et 253) dans Richard III, lorsque Macbeth affirme qu'il a donné le joyau éternel de son âme à «l'ennemi commun des hommes » (" the common enemy of man » III.i.67-8) ou encore quand Viola mentionne «The pregnant enemy» («l'ingénieux 
ennemi » II.ii.28) dans La Nuit des Rois, le public élisabéthain pouvait identifier sans peine celui à qui il était fait allusion et dont le nom même, Satan, signifie d'ailleurs l'ennemi. Ce personnage était très présent dans les esprits et son activité de collecteur d'âmes de choix - Méphistophélès est en quête de "glorious souls » dans Dr Faustus - était suffisamment connue pour faire l'objet de métaphores audacieuses. Ainsi, lorsqu'Olivia, la comtesse d'Illyrie, affirme: «A fiend like thee might take my soul to hell » (III.iv.212), le dramaturge ne ressent pas le besoin d'expliciter son propos. De même, un grand nombre de personnages sont comparés au diable: c'est le cas, par exemple, de Shylock dans Le Marchand de Venise - «the Jew is the very devil incarnation » (II.ii.26) -, de Prospéro dans La Tempête - «The devil speaks in him» (V.i.129) dit Sebastian -, de Macbeth dont la cruauté surpasse celle des légions infernales (Macduff, IV.iii.55-7), de Richard Gloucester dans Richard III ou encore d'Angelo dans Mesure pour Mesure. (V.i.30)

Diable n'a acquis la popularité que lui confèrent les personnages de Shakespeare qu'au terme d'une lente évolution. Robert Muchembled $^{2}$ a démontré que ce processus a été accompagné par le développement et la cristallisation de son apparence. Au Moyen-Âge, il revêtait des formes multiples : il combinait entre autres les attributs du bouc, du chien, du lion, du serpent, du dragon et de l'ours et renvoyait à des traditions multiples. On peignait sa peau en rouge, en noir ou en vert ${ }^{3}$. Le diable était aussi parfois représenté avec deux visages, ce qui attestait son caractère vorace et insatiable. C'était une créature multiple et ce manque d'unité était un obstacle à l'émergence d'une peur profonde. Ce diable hybride n'était pas très inquiétant. On pensait pouvoir facilement le berner et le renvoyer en enfer comme le suggère Dumaine qui, dans Peines d'Amour Perdues, est en quête de stratagèmes pour le tromper : «Some tricks, some quillets, how to cheat the devil.» (IV.iii.279)

4 L'auteur d'Une Histoire du Diable, XIIème-XXème siècle affirme que « jusqu'au XIIème siècle, le monde était trop enchanté pour permettre à Lucifer d'occuper tout l'espace de la peur, de la crainte et de l'angoisse ${ }^{4}$.» Pour revigorer la foi déclinante et, comme le dit Roland Villeneuve, "déraciner le paganisme dans les cœurs et les esprits ${ }^{5}$ ", l'Église devait brandir un épouvantail véritablement terrifiant. Toutes les peurs devaient être canalisées et associées à une seule entité. Les autres créatures - lutins, gobelins, satyres et autres méchants de tous poils - allaient devoir s'effacer devant l'Ennemi de Dieu officiel et son armée de sorcières. Un ange inversé a été élaboré : les ailes colorées sont devenues des ailes de chauve-souris, son auréole a cédé la place au cercle interrompu d'une paire de cornes, et sa beauté s'est putréfiée en laideur immonde. La laideur de Satan est inversement proportionnelle à la beauté de l'ange qu'il fut. Ce qui fait peur dans son apparence c'est le fantôme immanent d'une beauté perdue ${ }^{6}$. Le Sonnet 94 de Shakespeare n'affirme-t-il pas que, quand ils pourrissent, les lys sentent plus mauvais que les mauvaises herbes? («Lilies that fester smell far worse than weeds.») La beauté parfaite de l'ange s'est transformée en laideur ultime qui a, à son tour, suscité une peur sans fond ; sans fond, comme l'enfer, ce lieu de suppliques que chacun imagine et auquel les peintres donnent un visage lui aussi identifiable.

On peut résumer cette stratégie ecclésiastique en disant qu'un loup a été créé afin que les moutons apeurés se tournent à nouveau vers le berger pour obtenir sa protection. La peur est donc au cœur de cette manœuvre. Le Diable en est devenu l'incarnation, une visibilité qui «nous regarde et peut nous engloutir ${ }^{7}$ ». Le règne de la terreur, que la philosophe appelle " phobocratie $e^{8}$ " pouvait s'affirmer et les regards régresser vers les craintes de l'enfance puisque, comme l'affirme Lady Macbeth, «'Tis the eye of childhood / That fears 
a painted devil. » (II.ii.53-4) L'élaboration de la figure centrale du Diable à partir de traditions multiples s'accompagne d'un mouvement régressif visant à fragiliser les individus. La peur du diable est donc une création politique, au sens large du terme. La place majeure qu'il a occupée dans les esprits à la Renaissance indique que cette période ne fut une libération que pour quelques uns, comme le souligne Jean Delumeau dans $L a$ Peur en Occident, « mais (que) pour la plupart des membres de l'élite européenne, elle fut sentiment de faiblesse ${ }^{9}$. » Le royaume du Prince des Ténèbres s'étendait à l'infini au delà de la mort et la croyance en son existence devint, selon Christopher Hill, l'un des instruments de persécution religieuse les plus efficaces ${ }^{10}$. Les hommes ont pris la mesure de leur petitesse, petitesse que la mer leur avait déjà signifiée.

\section{La mer}

6 Dans l'ouvrage qu'il a consacré à la peur, Jean Delumeau affirme que " pour le plus grand nombre, (la mer) est restée longtemps dissuasion et par excellence le lieu de la peur ${ }^{11}$.» "Si tu veux apprendre à prier, va sur la mer" dit un proverbe portugais. La scène d'exposition de La Tempête de Shakespeare est donc une scène particulièrement terrifiante: les spectateurs assistent impuissants au naufrage d'un bateau que les eaux engloutissent avec tout son équipage. La mer dévorante est aveugle et rien ne peut calmer ses ardeurs, pas même le nom de roi. La mort qui attend les marins est terrifiante et Gonzalo lui préfèrerait toute autre alternative. Les derniers mots qu'il prononce tandis que le navire s'enfonce lentement dans les entrailles froides des flots sont empreints d'une terrifiante résignation :

GONZALO. Now would I give a thousand furlongs of sea for an acre of barren ground

- long heath, brown furze, anything. The wills above be done, but I would fain die a dry death.

L'allitération finale en $\mathrm{D}$ nous permet d'entrer dans la poitrine du personnage et d'entendre les derniers battements de son cœur. Cet homme qui sombre partage la peur du maître d'équipage qui demande si leurs bouches sont condamnées au froid : «What, must our mouths be cold? " " Mourir, » la belle affaire, semblent-ils dire, mais devenir froid, sentir que la chaleur de ce courant interne va devenir une motte de terre compacte est insupportable. C'est ce que dit Claudio depuis sa cellule dans Mesure pour Mesure. ( "This sensible warm motion to become / A kneaded clod» III.i.119) Dans un élan poétique, Shakespeare fait du cadavre une clef froide et lourde : « Key-cold figure of a holy king, » (I.ii.5) dit Lady Anne en touchant le cadavre de son beau-père. Le trépas peut être décrit comme la perte de la chaleur de la vie. Les profondeurs glacées de la mer lui sont tout naturellement associées car elles prennent possession des corps qui les atteignent. Le passage est rapide et pour signifier que le naufrage vient tout juste de se produire, le clown du Conte d'Hiver déclare que les corps des marins ne sont pas encore froids : «the men are not yet cold under water.» (III.iii.104) Les flammes de l'enfer sont presque rassurantes à côté de la sépulture glacée des flots. Homère le savait bien, lui dont Sebastian Brant affirma en 1494 dans sa Nef des Fous qu'il « inventa l'histoire d'Ulysse pour nous apprendre à être sages, à craindre l'aventure en mer $^{12}$. " Sébastian Brant lui-même le savait bien : la vie est un naufrage et les hommes des fous destinés aux vagues dévorantes (345):

Cherchons fortune en ce marasme

Où l'on ne peut que s'échouer.

Puis rompent mats, voiles et cordes, 
À peine pouvons nous flotter

La vague est haute comme un mont,

Les hommes ne savent plus bien

où est le ciel, où est le fond.

(...)

Sommes perdus, désemparés

Et dans le gouffre allons sombrer.

8 La mer avale ceux qu'elle éloigne de la «terre mère ». Elle devient un monstre hideux dont les bateaux gravissent la crinière monstrueuse. Dans Othello, un gentilhomme décrit les montagnes d'eau qui se dressent jusqu'aux cieux puis se déversent sur les embarcations (II.i.12-15) :

SECOND GENTLEMAN. The chiding billow seems to pelt the clouds,

The wind-shak'd surge, with high and monstrous main,

Seems to cast water on the burning bear,

And quench the guards of the ever-fixed pole

Le naufrage en mer fait échapper les marins aux regards des observateurs que ce spectacle emplit d'effroi. C'est ce que démontre la scène rapportée dans Le Conte d'Hiver après que le navire d'Antigonus a été avalé par les flots cruels (III.iii.97-99) :

CLOWN. But to make an end of the ship, to see how the sea flap-dragoned

it: but first, how the poor souls roared, and the sea mocked them

La tombe glacée des eaux profondes fait donc peur aussi car elle fait disparaître ceux dont elle ne restitue pas les corps froids. Ainsi, les flots se referment sur les hommes sans laisser une trace de leur passage. La mer est une tombe muette - « watery grave » (Périclès , II.i.10) - sur laquelle on ne peut pas graver son histoire. Il ne reste que l'absence et la présence silencieuse de « simples coquillages. » (Périclès, III.i.69)

\section{L'enfer sous-marin}

11 La mer est un monstre indomptable et imprévisible qui dévore les hommes et, ce faisant, ravive le souvenir du Déluge biblique. Elle peut atteindre les cieux et descendre jusqu'aux tréfonds de la terre qu'elle recouvre. Ainsi, en passant de l'un de ces extrêmes à l'autre, elle fait le lien entre le paradis et l'enfer. C'est ce que suggère Othello lorsqu'il évoque une embarcation malmenée par les vagues (II.i.187-9) :

OTHELLO. And let the labouring bark climb the seas,

Olympus-high, and duck again as low

As hell's from heaven.

12 Puisque les neuf cercles de l'enfer décrits par Dante atteignent le centre de la terre, il est possible de penser que le fond des océans permet d'atteindre ce lieu redouté. Après tout, la mer et l'enfer ont en commun la profondeur qu'Isabella évoque dans Mesure pour Mesure lorsqu'elle décrit le fourbe Angelo (III.i.92-3):

ISABELLA. His filth within being cast, he would appear

A pond as deep as hell.

13 Cependant, bien que les Saintes Écritures fassent allusion à un puits sans fond («the bottomless pit »), Thomas Hobbes rappelle à ses lecteurs que la profondeur de l'enfer ne peut pas être réellement infinie car la terre a une circonférence ${ }^{13}$. Même si les descriptions des tourments de l'enfer que proposent les personnages de Shakespeare mettent en avant la souffrance des damnés soumis au feu ou à la glace, l'évocation de ce 
lieu comporte parfois une allusion à l'élément liquide. Claudio, qui souhaite convaincre sa sœur de sacrifier au Duc les trésors de son corps, évoque des flots ardents (III.i.116-128) :

CLAUDIO. Ay, but to die, and go we know not where;

To lie in cold obstruction and to rot;

This sensible warm motion to become

A kneaded clod; and the delighted spirit

To bathe in fiery floods, or to reside

In thrilling region of thick-ribbed ice;

To be imprison'd in the viewless winds,

And blown with restless violence round about

The pendent world; or to be worse than worst

of those that lawless and incertain thought

Imagine howling: 'tis too horrible!

The weariest and most loathed worldly life

That age, ache, penury and imprisonment

Can lay on nature is a paradise

To what we fear of death.

Sa description rappelle le second cercle de l'Enfer de Dante où le vent fouette les damnés, les septième et huitième cercles où les âmes sont immergées dans du sang bouillant ou des lacs de feu, et le neuvième où elles sont en parties figées dans des blocs de glace. Quant à Othello il estime mériter d'être immergé dans des fleuves de lave. (V.ii.278-281) ${ }^{14}$ Il est vrai que l'enfer chrétien est parcouru par des fleuves hérités de la tradition grecque : le Styx, l'Achéron empoisonné que Charon permet de traverser, le Léthé, le Phlégéthon (le fleuve de feu) et le Cocyte (le fleuve glacé des lamentations). Thomas Hobbes rattache d'ailleurs timidement le mot Hell au grec Hadès. ${ }^{15}$

Les descriptions proposées par Othello ou Claudio sont conventionnelles et renvoient au texte de Dante. Associer le fond de la mer à l'enfer, comme le fait Shakespeare dans Richard III, est peu orthodoxe. En effet, lorsque les poètes et les dramaturges anglais de la Renaissance imaginaient le fond des mers, ce qu'ils décrivaient était le plus souvent merveilleux. Ainsi, dans Hero and Leander, Christopher Marlowe campe le paysage suivant :

17 Leander striv'd, the waves about him wound, And pull'd him to the bottom, where the ground Was strew'd with pearl, and in low coral groves Sweet singing mermaids sported with their loves On heaps of heavy gold, and took great pleasure To spurn in careless sort the shipwrack treasure.

$18 \mathrm{Au}$ fond de l'eau se trouvent les belles sirènes amoureuses, le palais d'azur de Neptune et les trésors engloutis que le dieu entend offrir à Léandre : « to the rich Ocean for gifts he flies. » Dans Henry V, Canterbury évoque le fond de la mer avec ses épaves et ses trésors innombrables (I.ii.164-5). La chanson d'Ariel dans La Tempête décrit les coraux que sont devenus les os du père de Ferdinand (I.ii.397-403) :

ARIEL. Full fathom five thy father lies,

of his bones are coral made;

Those are pearls that were his eyes,

Nothing of him that doth not fade

But doth suffer a sea-change 
Into something rich and strange

Sea nymphs hourly ring his knell. vision est qu'elle conjugue la peur de la mer et la peur de l'Enfer. Déjà, au début de $L a$ Tempête, Ariel avait rapproché l'enfer de la mer en rapportant les propos de Ferdinand selon qui tous les démons de l'enfer s'étaient donné rendez-vous sur le bateau : «Hell is empty, And all the devils are here. » (I.ii.214-5). Ce lien avait été également suggéré par le Malleus Maleficatum puisque c'est en les jetant dans l'eau - même en la présence de leurs parents - que les sorcières s'appropriaient les âmes des jeunes enfants qu'elles ne dévoraient pas $^{16}$. Bien que la Bible mentionne à deux reprises (Job, 26:5 et Isaïe, 14:9) la prison sous-marine des géants, comme le rappelle Thomas Hobbes dans son Léviathan ${ }^{17}$, ce qui se passe après la mort est souvent représenté comme une descente sous la terre. De nombreuses visions eschatologiques sont évoquées en littérature et le mythe d'Er au livre $\mathrm{X}$ de la République de Platon en est un exemple célèbre. Au début de La Tragédie Espagnole de Thomas Kyd le fantôme d'Andréa arrive directement sur la rive de l'Achéron ${ }^{18}$. Dans le livre IV des Métamorphoses, Ovide décrit un chemin sinueux qui descend vers le Styx. Quant à Dante, il passe par la porte de l'enfer dont la dernière ligne a marqué les esprits : "Lasciate ogne speranza, voi ch'intrate." L'approche que propose shakespeare dans Richard III est unique en ce sens qu'elle permet d'assister à la mort du personnage et à son passage dans l'autre monde.

21 Tout d'abord, Clarence rêve qu'il traverse la Manche en bateau avec son frère Richard pour se rendre en Bourgogne (I.iv.10). Les deux hommes se bousculent et Clarence tombe par-dessus bord. Il est englouti et ses yeux restent ouverts tout au long de sa descente. Il faut dire que le prisonnier rêve une mort par noyade particulièrement terrifiante et douloureuse : « O Lord! Methought what pain it was to drown. » (I.iv.21) La vision est ici moins paisible que dans la chanson d'Ariel (24-33):

CLARENCE. Methought I saw a thousand fearful wrecks;

Ten thousand men that fishes gnaw'd upon;

Wedges of gold, great anchors, heaps of pearl,

Inestimable stones, unvalu'd jewels,

All scatter'd in the bottom of the sea.

Some lay in dead men's skulls, and in the holes

Where eyes did once inhabit, there were crept -

As 'twere in scorn of eyes - reflecting gems,

Which woo'd the slimy bottom of the deep,

And mock'd the dead bones that lay scatter'd by.

Les perles d'Ariel ont été remplacées par des diamants nichés dans les orbites des crânes, les naufragés sont mangés par les poissons et les os jonchent le sol. Malgré la présence des joyaux, le fond de la mer est sale et inhospitalier. C'est une «mort boueuse ${ }^{19}$ que la noyade entraîne. La description effectuée par Clarence rappelle la caverne de Mammon que Guyon visite dans le chant 7 du livre II de The Fairie Queen d'Edmund Spenser ${ }^{20}$. Les mots de Lady Macbeth nous reviennent à l'esprit. "L'enfer est poisseux », (" Hell is murky » V.i.34) avait-elle dit. La descente de Clarence s'accompagne de l'emprisonnement de l'âme dans le corps mourant du rêveur, les flots jaloux refusant de la laisser rejoindre les airs. Alors commence la tempête dans son âme (44) qui annonce le début du voyage dans l'au-delà, phase que Thomas Hobbes appelle «la deuxième mort " ${ }^{21}$. Les couleurs 
disparaissent. Ensuite, Clarence traverse l'Achéron sur l'embarcation de Charon et atteint le pays de la nuit éternelle (47) qui semble correspondre à son arrivée dans les profondeurs abyssales. Là il rencontre les âmes de ses victimes qui lui rappellent sa traitrise et envoient des démons le tourmenter. Leurs cris terribles le réveillent et pendant un moment encore il pense être en enfer.

Clarence se retrouve en enfer car il a été bousculé par le Diable. C'est du moins le rôle que se donne Richard dans le rêve éveillé de sa quête : «(I) seem a saint when most I play the devil » dit-il (I.iv.338). Dans 3 Henry VI, Richard Gloucester avait dit son ambition folle. Il avait annoncé son intention d'assécher la mer pour atteindre la couronne qui brillait de l'autre côté des flots. Puis il avait ajouté qu'il vivrait en enfer tant qu'il n'y serait pas arrivé (III.ii.146-171) :

RICHARD. I'll make my heaven to dream upon the crown,

And, whiles I live, to account this world but hell,

C'est donc un monde aquatique, un enfer sous-marin qu'il entend assécher, que Richard évoque. Dans Richard III, la mère du héros déclare qu'il a fait de ce monde un enfer pour elle («Thou camest on earth to make the earth my hell. » IV.iv.167-8) et son épouse affirme qu'il ne sera à sa place qu'en enfer (I.ii.111). Le monstre a donc atteint son but! La pièce Richard III peut être considérée comme la réalisation du rêve cauchemardesque que le héros a annoncé dans 3 Henry VI. Le spectateur attentif peut même se surprendre à penser que la pièce Richard III a été rêvée par 3 Henry VI. Au début de la représentation de Richard III, les spectateurs de théâtre s'endorment, comme Clarence ${ }^{22}$ dans le rêve terrifiant du monstre. Le passage de la réalité au rêve est effectué grâce au discours hypnotique initial prononcé par Richard qui pénètre leurs esprits à grands coups de "Now» et de "I ». La scène d'introduction peut d'ailleurs être perçue comme une tentative du personnage malfaisant de prendre possession de l'esprit et du regard des spectateurs. Lui qui est visible sur la scène se décrit pourtant et il renchérit sur ses malformations. C'est son regard déformant qu'il entend substituer à celui des spectateurs à qui il impose une vision anamorphique. Il essaiera finalement de s'enfuir à cheval après avoir transformé le monde en abattoir, en cauchemar, en night-mare. Quitter le cauchemar à cheval, belle ironie. Mais Richard ne sait pas qu'on ne peut pas s'extirper d'un cauchemar: Jorge Luis Borges nous rappelle que c'est le cauchemar qui nous chasse en nous infligeant une surdose de peur. Des gouttes de sueur glacées peuvent perler sur son front: son ennemi, Richmond, déclare se battre aux côtés de Dieu («God, and our good cause, fight upon our side. » V.iii.240) et il va chasser l'apprenti diable de la pièce.

\section{Conclusion}

Associer l'enfer et la mer, comme le fait Shakespeare dans Richard III, c'est mêler l'angoisse de ce que Jankelevitch appelle «le terrible seuil du mourir ${ }^{23}$ » et la peur des tourments infernaux qu'orchestre le diable. C'est aussi symboliquement placer l'enfer dans le royaume de Dieu : en effet, dans The Great Code, Northrop Frye nous rappelle que "sous l'un des aspects du symbolisme, les eaux du Déluge ne se sont jamais retirées et nous sommes tous des poissons dans un monde d'illusions qui est symboliquement sousmarin ${ }^{24}$.» Une fois qu'il aura asséché la mer, Richard sera parvenu à éliminer le monde aquatique divin pour le remplacer par l'enfer qu'il porte en lui. Et cet enfer sera son paradis. 
Dans Richard III, la crainte du passage se conjugue à la terreur que suscite l'idée d'une éternité de douleurs. La peur n'est pas causée par le fait que l'on ne sait pas où l'on va, comme le dit Claudio, mais plutôt par la certitude du châtiment que l'on mérite. Finalement, l'enfer est en chacun d'entre nous et c'est notre conscience qui lui ouvre les portes. En 1636, Thomas Browne affirmera dans son Religio Medici : "The heart of man is the place the devil dwells in; I feele sometimes a hell within my selfe, Lucifer keeps his court in my breast ${ }^{25}$. »Clarence porte donc l'enfer en lui et il l'entraîne au fond des mers. Il pourrait dire, comme Méphistophélès dans le Doctor Faustus de Christopher Marlowe, que l'enfer est le lieu où le damné se trouve («Where we are is hell », 2.i.122). Le rêve de Clarence est donc intime, puisque le personnage rencontre les âmes de ceux qu'il a assassinés, mais il est aussi collectif car il se nourrit des craintes communes à tous les hommes. "Rien n'est plus difficile à analyser que la peur, dit Jean Delumeau, et la difficulté s'accroitt encore lorsqu'il s'agit de passer de l'individuel au collectif ${ }^{26}$. " Le théâtre permet ce glissement de l'un au multiple puisque chacun est isolé du public qu'il constitue par sa réception du spectacle. Le dramaturge a su capter les inquiétudes de son temps et mettre en scène les cauchemars de ses contemporains. Si le cauchemar est le lieu de la peur ultime, de la peur la plus profondément ensevelie, sa représentation et son partage grâce à la représentation théâtrale ne sont-ils pas le moyen de glisser de l'individuel au collectif ? Les mots de Jorge Borges pourraient terminer cet article: « Les cauchemars seraient-ils des brèches de l'enfer? Serions-nous dans le cauchemar, littéralement en enfer? Pourquoi pas? Tout est si étrange que même cela est possible ${ }^{27}$.

\section{NOTES}

1. Les références aux pièces de Shakespeare sont celles des éditions Arden: The Arden Shakespeare Complete Works, éd. Richard Proudfoot, Ann Thompson et David Scott Kastan, Londres et New York, Bloomsbury, 2011.

2. Robert Muchembled, Une Histoire du Diable XII'-XX' siècle, Paris, Seuil, 2000.

3. Michel Pastoureau signale que certains diables étaient peints en bleu. Les importateurs et les marchands de garance qui avaient peur de la dangereuse concurrence du bleu dans les pratiques vestimentaires ont payé des artisans pour qu'ils représentent des diables bleus dans les vitraux...

4. Robert Muchembled, Une Histoire du Diable, XIIe-XXe siècle, Paris, Seuil, 2000, p. 33.

5. Roland Villeneuve, La Beauté du Diable, Paris, Bordas, 1994, p. 31.

6. Cette beauté était parfaite, ce qui implique qu'elle recouvrait le masculin et le féminin comme l'indique Méphistophélès dans le Faust de Marlowe :

"I'll cull thee out the fairest courtezans, / And bring them every morning to thy bed: / She whom thine eye shall like, thy heart shall have, / Were she as chaste as was Penelope, / As wise as Saba, or as beautiful / As was bright Lucifer before his fall."

7. Marie José Mondzain, L'Image peut-elle tuer ? Paris, Bayard, 2002, p. 28.

8. Marie José Mondzain, Homo Spectator, Paris, Bayard, 2007, p.73.

9. Jean Delumeau, La Peur en Occident, Paris, Fayard, 1978, p. 331. 
10. Voir Christopher Hill, The World Turned Upside Down: radical ideas during the English reformation, Harmondsworth, Penguin Books, 1972, p. 177-8.

11. Delumeau, op. cit. p. 39.

12. Sebastian Brant, la Nef des Fous, Paris, José Corti, 1997 (1494), p. 344.

13. "Not that the Writers of the Scriptures would have us beleeve, there could be in the globe of the Earth, which is not only finite but also (compared to the height of the Stars) of no considerable magnitude, a pit without a bottome; that is, a hole of infinite depth, such a the Greeks in their Demonologie and after them in the Romans called Tatarus (...) for that is a thing the proportions of Earth to Heaven cannot bear: but that wee should beleeve them there, indefinitely, where those men are, on whom God inflicted that Exemplary punishment.» Leviathan, Part III, Chapter 38, London, Penguin Classics, 1985 (1651), p. 485-6.

14. « Whip me, ye devils, / From the possession of this heavenly sight! / Blow me about in winds! Roast me in sulphur! / Wash me in steep-down gulfs of liquid fire! »

15. « Hades (from which word perhaps our word Hell is derived). » Leviathan, London, Penguin Books, 1987 (1651), p. 487.

16. Henry Institoris et Jacques Sprenger, Malleus Maleficatum (1486), Paris, Jérome Million, 1997, p. 276. «Des enfants marchant au bord de l'eau, elles savent sans se faire voir les jeter dans l'eau en présence même des parents. »

17. Leviathan, p. 486.

18. «When I was slain, my soul descended straight / To pass the flowing Stream of Acheron.» $(\mathrm{I}, 1,18-19)$

19. C'est ainsi qu'est décrite la mort par noyade d'Ophélie dans Hamlet : «But long it could not be / Till that her garments, heavy with their drink, / Pull'd the poor wretch from her melodious lay / To muddy death. » (IV,7, 179-182)

20. «But all the ground with skulls was scattered, / And dead mens bones, which round about were flung / Whose lives, it seemed, whilome there were shed, / And their vile carcases now left unburied. » Spenser, The Faerie Queene, London and New York, Longman, 1984 (1590), p. 229.

21. Leviathan, op. cit., p. 490.

22. On notera que Dante s'endort lui aussi en arrivant au bord du fleuve Achéron : «De cette terre de larmes jaillit un souffle, qui projeta un éclair de feu, lequel m'enleva tout sentiment ; / et je tombai comme un homme qui succombe au sommeil. » Dante, La Divine comédie, (publ. 1472), trad. D’Alexandre Masseron, Paris, Albin Michel, 1995, p. 77.

23. Vladimir Jankelevitch, La Mort, Paris, Flammarion, 1977, p. 65.

24. Northrop Frye, The Great Code: la Bible et la littérature, Paris, Seuil, 1982, p. 210.

25. Thomas Browne, Religio Medici, 1636, section 51.

26. La Peur en Occident, p. 26.

27. Jorge Luis Borges, Conférences, Paris, Gallimard, 1980, p. 53.

\section{RÉSUMÉS}

Dans La Peur en Occident, Jean Delumeau affirme que la mer est le lieu des peurs les plus profondes. Mais s'il en est ainsi, c'est que la "grande verte" est le lieu d'une mort particulièrement inquiétante: la noyade. Même disloqué, le corps du supplicié est rendu à la terre mais avant de mourir, le naufragé est emporté par les flots puis happé par les profondeurs 
marines. Là se produit le passage. Le noyé échappe aux regards et sa mort devient absence. C'est pour cela que dans La Tempête Gonzalo aspire à une "mort sèche " ( "I would fain die a dry death» I,1,67). Le danger et l'imprévisibilité des flots sont mentionnés plusieurs fois dans le théâtre de Shakespeare et ils sont un ressort dramatique (Le Marchand de Venise, Péricles, La Nuit des Rois). Mais la crainte des courants qui happent le naufragé est aussi liée à la présence d'un monde sous-marin et secret dont Clarence a la révélation dans un rêve. Richard III contient la description hallucinée de ce lieu qu'habitent les démons et où les nuages sont ensevelis (I,1,3-5). Le Diable, l'ennemi commun des hommes qu'on avait alors tendance à représenter dans un lieu fermé et souterrain, y a donc lui aussi élu domicile. Il est partout, même dans les profondeurs froides de la mer. L'enfer qu'évoque Claudio dans Mesure pour Mesure est presque rassurant face à celui que peint Clarence. C'est un lieu que Dante a décrit et dont la topographie était connue à l'époque. La terreur que suscite l'enfer du malheureux frère de Richard Gloucester est plus terrifiante car elle déplace une peur dans une autre. C'est la peur ultime.

In La Peur en Occident, Jean Delumeau asserts that the deepest fears are related to the sea. This can be explained by the fact that the "big green" can cause people to drown and drowning is a particularly worrying form of death. Even the criminal's dismembered body is given back to the earth; but the castaway's is carried away by the floods then swallowed by the watery depths until the final passage occurs. The drowning sailor disappears and his death boils down to an absence. This is why, at the beginning of The Tempest, Gonzalo wishes for a "dry death." («I would fain die a dry death » 1,1,67). Shakespeare's drama contains many allusions to the unpredictable and dangerous seas that serve as dramatic engines (The Merchant of Venice, Pericles, Twelfth Night). Yet the fear of the floods that carry the castaway goes hand in hand with the presence of a secret underwater world that Clarence glimpses in his dream. In Richard III, this amazing world, inhabited by devils and where the threatening clouds were buried (1,1,3-5), is described by the dreamer. The Devil, the common enemy of man who was usually represented confined to the nether world, seems to haunt this place. He is everywhere to be found, even in the deep cold sea and Claudio's depiction of Hell in Measure for Measure may look reassuring when compared with Clarence's vision. The former refers to Dante's description of it and it was familiar to Renaissance minds. The terror raised by Richard's poor brother's description is all the more bloodcurdling as it causes a fear to be hosted by another one. It is the ultimate fear.

\section{INDEX}

Keywords : Clarence, death, devil, dream, drowning, Gloucester Richard, nightmare, sea, treasure.

Mots-clés : cauchemar, Clarence, diable, Gloucester Richard, mer, mort, noyade, rêve, trésor

\section{AUTEUR}

JEAN-LOUIS CLARET

Université d'Aix-Marseille, LERMA. 